\title{
editorial
}

\section{Finding good quality information}

\section{Derek Richards}

\author{
Editor
}

Evidence-Based Dentistry (2002) 3, 1. DOI: 10.1038/sj/ebd/6400097

Welcome to the third volume of Evidence-based Dentistry. The last year has seen a variety of problems: my own illness has seen me unable to work for a period of almost 4 months which, combined with staff changes at our publishers, has led to a series of delays in the publication of the journal. For those of you who eagerly await our publication to drop through the door I am sorry. We hope that these delays are now firmly behind us and that we will continue to go from strength to strength. The new cover design is one tangible sign of this new approach and we are in the process of defining a new internal style for the journal.

Whether you are the editor of a journal or a busy practitioner one thing that you need to access is good-quality information. One of my challenges in running this journal of secondary publication is the identification of goodquality information from the wealth of dental journals that are available worldwide. To date we have been relying on a committed team of individuals who seek to identify articles that meet our criteria for inclusion in the journal. Although they have performed admirably over the early years of the journal we are now moving towards a more systematic approach more in keeping with the methodology of an evidencebased approach. It is to this end that we have published a primary research article for the first time. The article, entitled 'Benchmarking the dental randomised controlled trial literature on MEDLINE', provides a systematic examination of the available dental literature on the most readily available source of information, ie, MEDLINE. It also looks at a range of specific searches for dental specialities. Another first for the journal is that because of space constraints we have published the Appendix tables, containing the detailed search strategies, for the specialities on the journal's website (www.nature.com/ebd).

Another reason for publishing this article is that these search strategies will form the basis for our regular searches of the dental literature to provide material for inclusion in Evidence-based Dentistry. This will extend our scanning of the dental literature beyond the 50 top dental journals that we have been restricted to previously with the old system of using 'spotters'. The one downside of this approach is that it restricts our vision to those dental journals that are indexed on MEDLINE. Although this covers a major propor- tion of the 700-plus dental journals available world-wide, it does put us in the position of possibly missing an important article so we will still be working with our spotters and any readers to ensure that we do not miss any high quality relevant articles. If you wish to contribute please let the editor know via e-mail (ebdeditor@nature. com).

In this issue we have looked at the range of questions covered in the first two volumes of Evidence-based Dentistry. We also have abstracts from two of the latest Cochrane systematic reviews published in the Cochrane library by the Cochrane Oral Health Group - as well as the usual mix of summaries of published papers and digests from other evidence-based publications.

The Centre for Evidence-based Dentistry in Oxford contributes much to the production of this journal, and as one of its goals is to raise the profile of evidence-based dentistry we welcome the appearance of the journal of Evidence-based Dental Practice in the USA. This journal has a slightly different focus to our own but it does show that the interest and demand for more information about the evidence-based approach in dentistry is growing. 